\title{
A survey of the attitudes and beliefs about the use of TENS for pain management by physiotherapists working in two cities in Sri Lanka
}

This article was published in the following Dove Press journal:

Patient Related Outcome Measures

14 May 2014

Number of times this article has been viewed

\author{
Thusharika D Dissanayaka' \\ Gourav Banerjee 2,3 \\ Mark I Johnson ${ }^{2,3}$ \\ 'Department of Physiotherapy, Faculty \\ of Allied Health Sciences, University \\ of Peradeniya, Peradeniya, Sri Lanka; \\ ${ }^{2}$ Centre for Pain Research, Faculty \\ of Health and Social Sciences, Leeds \\ Metropolitan University, Leeds, UK; \\ ${ }^{3}$ Leeds Pallium Research Group, Leeds, \\ UK
}

Introduction: Transcutaneous electrical nerve stimulation (TENS) is a noninvasive, inexpensive, self-administered technique used throughout the world to relieve pain. In Sri Lanka, physiotherapists may use TENS for their patients as they receive a small amount of education about the principles and practice of TENS in their undergraduate training. To date, there have been no data gathered about the use of TENS by physiotherapists in Sri Lanka. The aim of this study was to assess attitudes and beliefs of physiotherapists working in Sri Lanka about their use of TENS for pain management.

Methods: A postal survey was undertaken using a 12-item questionnaire developed by the investigators to gather information about attitudes, beliefs and use of TENS in clinical practice. The questionnaire was distributed to 100 physiotherapists working in three government hospitals and six private hospitals in the cities of Kandy and Colombo. A descriptive analysis of data was performed.

Results: Sixty-seven completed questionnaires were returned (67\% response rate). Over half of the respondents $(58.2 \%)$ reported that they used TENS to treat pain "often" or "very often", with use for musculoskeletal/orthopedic (61.3\%) and neuropathic/neuralgic (79.1\%) pain being most common. TENS was used less for postsurgical pain and rarely for cancer pain. Most (95.5\%) respondents reported that their patients benefitted "considerably" from TENS. $76.1 \%$ of the respondents reported that they did not recommend and/or prescribe TENS for patients to use at home.

Conclusion: Physiotherapists value TENS as a treatment option to manage musculoskeletal and neuropathic pain. However, there is a need for systems and resources to enable to patients to self-administer TENS rather than having to visit clinics.

Keywords: transcutaneous electric nerve stimulation, electrotherapy, non pharmacological analgesia

\section{Introduction}

The use of electricity to manage human ailments is an age-old concept that dates back to $2500 \mathrm{BC}$ when ancient Egyptians used electric fish to produce "shocks" to relieve pain. ${ }^{1}$ During the 19 th century, the use of electricity in medicine gained popularity with the development of a variety of electromedical devices. From the mid-1960s, the pioneering works of Melzack and Wall, ${ }^{2}$ Shealy et al, ${ }^{3}$ Reynolds, ${ }^{4}$ and Long $^{5}$ led to further developments in electroanalgesic techniques, resulting in the introduction of transcutaneous electrical nerve stimulation (TENS) into mainstream medicine.

TENS is a noninvasive, inexpensive, self-administered medical technique used throughout the world to relieve pain. ${ }^{6}$ The purpose of TENS is to excite low-threshold peripheral afferent nerve fibers that convey neural information related to non-noxious
Correspondence: Thusharika Dissanayaka Department of Physiotherapy, Faculty of Allied Health Sciences, University of Peradeniya, Augusta Hill, Sri Amarawansa Mawatha,

Peradeniya, Sri Lanka

Tel+948I 3999623

Email thushfhs@yahoo.com 
stimuli to the brain, as this has been shown to close the "pain gate" and relieve pain. ${ }^{7}$ During TENS, pulsed currents of varying amplitude, duration, and frequency are generated by a portable battery-operated device and delivered via lead wires to electrodes attached to the intact surface of skin (Figure 1). The electrodes may be self-adhesive or smeared with conducting gel and secured using touch fastener straps or medical tape. The electrical characteristics of TENS can be adjusted by the user and dosage titrated according to need whenever the individual is experiencing pain. For most patients, pain relief during TENS is rapid in onset and associated with few adverse effects or drug interactions. ${ }^{6-8}$ Systematic reviews and meta-analyses of randomized controlled clinical trials provide evidence that TENS is effective for relief of many types of pain, including musculoskeletal ${ }^{9}$ and postsurgical pain. ${ }^{10}$ Some systematic reviews have failed to find efficacy ${ }^{11}$ or have been inconclusive. ${ }^{12}$ This has been attributed to methodological shortcomings in randomized controlled clinical trials, including the use of inappropriate TENS technique leading to underdosing. ${ }^{13,14}$ In general, it is believed that TENS should be used as a standalone treatment for mild-to-moderate pain and as an adjunct to pharmacotherapy for moderate-to-severe pain. ${ }^{7,8}$ TENS has also been used in the management of dementia, ${ }^{15}$ postoperative nausea and vomiting, ${ }^{16}$ and wound healing. ${ }^{17}$

A recent systematic review revealed that the prevalence of chronic pain worldwide is high - over $25 \% .{ }^{18}$ There were insufficient reliable data by which to estimate with any certainty the prevalence of chronic pain in resource-limited countries with a Human Development Index (HDI) of less than 0.9 , although prevalence appeared to be similar to that seen worldwide. ${ }^{18}$ The prevalence of chronic pain in Sri

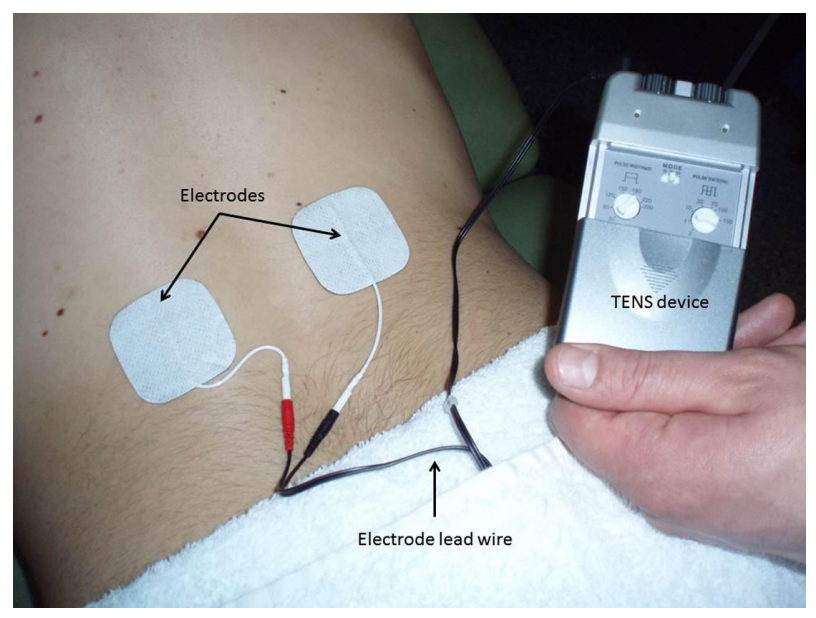

Figure I TENS device and accessories.

Abbreviation: TENS, transcutaneous electrical nerve stimulation.
Lanka has not been established. Census data from 2012 found that the national population was 20,263,723 (http://www. statistics.gov.1k/PopHouSat/CPH2012Visualization/htdocs/ index.php? usecase $=$ indicator\&action $=$ Map\&indId $=10$ ), ${ }^{19}$ so approximately 5.08 million people may be experiencing chronic pain at any one time if prevalence is taken as $25 \%$. This burden of pain is likely to have a major impact on national health care costs. ${ }^{20-22}$ Pharmacotherapy remains the mainstay treatment for painful conditions in developing countries including Sri Lanka, although analgesic medication may cause significant adverse effects and access to opioid medication is a problem. ${ }^{23}$ The financial costs of pharmacotherapy can be high, especially if patients are prescribed analgesics for prolonged lengths of time. ${ }^{23}$ For these reasons, good practice guidelines for pain management recommend nonpharmacological alternatives as adjuncts for the management of chronic pain.

In Sri Lanka, physicians, physiotherapists, nurses, and midwives may recommend the use of TENS to their patients. A TENS device and associated accessories are available without prescription and can be bought at pharmacy outlets or via the internet for approximately 20,000 Sri Lankan rupees. All physiotherapists receive a small amount of education about the principles and practice of TENS in their undergraduate training or during clinical practice. To date, there have been no data gathered about the use of TENS by physiotherapists in Sri Lanka. Therefore, the aim of this postal survey was to assess attitudes and beliefs of physiotherapists working in Sri Lanka about their use of TENS for pain management.

\section{Methods}

The study was approved by the ethical review committee, Faculty of Medicine, University of Peradeniya, Peradeniya, Sri Lanka. The postal survey was conducted throughout August and September 2012 on physiotherapists working in departments of physical medicine in government and private hospitals in two major Sri Lankan cities - Kandy and Colombo. At the time of the survey there were two government and two private hospitals with physiotherapy departments in Kandy, and two government and eleven private hospitals with physiotherapy departments in Colombo. Attempts were made to contact all physiotherapy departments to invite them to take part in the survey. Three government hospital departments (two in Kandy) and six private hospital departments (two in Kandy, four in Colombo) agreed to take part. The principal investigator (TDD) delivered, in person, a "survey pack" to each physiotherapy department.

The survey pack consisted of a participant information sheet, a formal invitation letter, and a survey questionnaire, 
and was given to all members of the physiotherapy team who managed mixed caseloads of "pain patients". The paper-based questionnaire consisted of 12 items and was designed by two members of the investigating team (GB, MIJ) and previously used on a population of Indian physiotherapists working in city hospitals in India. ${ }^{24}$ The questionnaire gathered information about the attitudes and beliefs of physiotherapists about their use of TENS and its role in pain management.

After 1 month, completed questionnaires were collected by departmental chief physiotherapists and sent via post to the principal investigator. Questionnaires were coded to maintain anonymity, although the location of the physiotherapy department was documented. A descriptive analysis was performed with data presented as counts and percentage responses to each question. It was decided not to conduct inferential statistics because of the relatively small sample size. SPSS v 17 (SPSS Inc., Chicago, IL, USA) was used to manage data and conduct the analysis.

\section{Results}

One hundred physiotherapists were given survey packs, and 67 completed questionnaires were returned $(67 \%$ response rate). Sixty of the $67(89.5 \%)$ respondents were registered physiotherapists, with seven of the $67(10.4 \%)$ respondents having their registration under renewal or pending (question [Q]12).

Of these 67 respondents, $64(95.5 \%)$ reported that they treated pain "very often" $(n=31)$ or "often" $(n=33)$ in their clinical practice $(\mathrm{Q} 1)$. Three respondents reported that they treated pain "occasionally". Thirty-nine $(58.2 \%)$ of these respondents reported that they used TENS to treat pain "often" (n=32) or "very often" (n=7) (Q2). Twenty-seven $(40.3 \%)$ respondents reported that they used TENS to treat pain "occasionally" and one respondent reported that they used TENS "seldom".

For the management of acute pain, $55.2 \%$ of respondents reported that they used TENS "often" or "very often", and $25.4 \%$ reported that they did not use TENS "at all" (Table 1 [Q3a]). For the management of subacute pain, $61.2 \%$ of respondents reported that they used TENS "often" or "very often", and only $6.0 \%$ reported that they did not use TENS "at all". For the management of chronic pain, 55.2\% of respondents reported that they used TENS "often" or "very often", and the percentage of respondents reporting not "at all" was zero.

Only 65 respondents provided answers for questions related to the severity of pain (Q3b). A larger proportion of respondents reported that they used TENS "very often" or "often" for pains that were more severe. For example, $23.1 \%$ of respondents reported that they used TENS "very often" or "often" for the management of mild pain, compared with $70.8 \%$ for the management of severe pain (Table 1 ). Only $12.3 \%$ of respondents reported that they did not use TENS "at all" for severe pain, compared with $29.2 \%$ for mild pain.

Respondents reported that they used TENS "very often" or "often" for pain associated with musculoskeletal/ orthopedics conditions $(61.3 \%)$ or neuropathies/neural-

Table I Responses to questions related to types and severity of pain

\begin{tabular}{|c|c|c|c|c|c|}
\hline \multirow[t]{2}{*}{ Pain types/conditions } & \multicolumn{5}{|c|}{ Respondents per response, $\mathbf{n}(\%)$} \\
\hline & Very often & Often & Occasionally & Seldom & Not at all \\
\hline \multicolumn{6}{|c|}{ Q3a: For which types of pain do you use TENS? } \\
\hline - Acute & $16(23.9)$ & $21(31.3)$ & $2(3.0)$ & II (I6.4) & $17(25.4)$ \\
\hline - Subacute & $6(8.95)$ & $35(52.2)$ & $14(20.9)$ & $8(11.9)$ & $4(6.0)$ \\
\hline - Chronic & $9(13.4)$ & $28(41.8)$ & $29(43.3)$ & $\mathrm{I}(\mathrm{I} .5)$ & $0(0)$ \\
\hline \multicolumn{6}{|c|}{ Q3b: For which types of pain do you use TENS? } \\
\hline - Mild & $6(9.2)$ & $9(13.85)$ & $23(35.4)$ & $8(12.3)$ & $19(29.2)$ \\
\hline - Mild to moderate & $8(12.3)$ & $8(12.3)$ & $25(38.5)$ & $19(29.2)$ & $5(7.7)$ \\
\hline - Moderate & $0(0)$ & $24(36.9)$ & $23(35.4)$ & $12(18.5)$ & $6(9.2)$ \\
\hline - Moderate to severe & $10(15.4)$ & $27(4 I .5)$ & $22(33.85)$ & $0(0)$ & $6(9.2)$ \\
\hline - Severe & $17(26.15)$ & $29(44.6)$ & II (I6.9) & $0(0)$ & $8(12.3)$ \\
\hline \multicolumn{6}{|c|}{ Q4: For which conditions do you commonly use TENS? } \\
\hline - Musculoskeletal/orthopedic & $21(31.3)$ & $20(29.85)$ & $15(22.4)$ & $4(6.0)$ & $7(10.45)$ \\
\hline - Neuropathy/neuralgia & $16(23.9)$ & $37(55.2)$ & $7(10.45)$ & $3(4.5)$ & $4(6.0)$ \\
\hline - Postsurgical & $8(11.9)$ & $6(8.95)$ & $26(38.8)$ & $16(23.9)$ & II (16.4) \\
\hline - Cancer pain & $0(0)$ & $\mathrm{I}(\mathrm{I} .5)$ & $4(6.0)$ & $15(22.4)$ & $47(70.1)$ \\
\hline - Other pains (eg, dysmenorrhea) & $0(0)$ & $0(0)$ & $10(14.9)$ & $22(32.8)$ & $35(52.2)$ \\
\hline
\end{tabular}

Note: There were 67 respondents for all questions except Q3b, for which there were 65 respondents. Abbreviation: TENS, transcutaneous electrical nerve stimulation. 
gias (79.1\%) (Table 1 [Q4]). Only $10.45 \%$ and $6.0 \%$ of respondents reported that they did not use TENS "at all" for musculoskeletal/orthopedic or neuropathic/neuralgia pain respectively. Fewer respondents reported that they used TENS "very often" or "often" for pain associated with postsurgical conditions $(20.9 \%)$ or cancer pain (1.5\%). Forty-seven (70.1\%) respondents reported that they that they did not use TENS "at all" for pain associated with cancer.

Sixty-four (95.5\%) respondents reported that their patients benefitted "considerably" from TENS, with two respondents reporting "No improvement noticed" (Q5). Fifty-one respondents $(76.1 \%)$ reported that they did not recommend/prescribe TENS for patients to use at home, suggesting that patients needed to make clinic visits to receive TENS treatment (Figure 2 [Q6]).

Twenty-seven (40.3\%) respondents reported that referring physicians had "never" recommended that the physiotherapist use TENS for pain relief (Figure 3 [Q7]), and 47.7\% of respondents reported that their patients had requested TENS treatment from them (Q8).

Twenty-five respondents (37.3\%) reported that their patients had used TENS before they came to receive pain treatment (Q9), and $44.8 \%$ of respondents reported that they believed that TENS was a cost-effective treatment option (Figure 4 [Q10]). Sixty-two respondents (92.5\%) reported that they also used TENS-like devices, including interferential therapy, to manage pain (Q11).

\section{Discussion}

This questionnaire survey of physiotherapists in two major cities in Sri Lanka found that $58.2 \%$ of respondents reported that they used TENS to treat pain "very often" or "often", although over 95\% reported that they believed that TENS was "considerably" beneficial. It was found that respondents

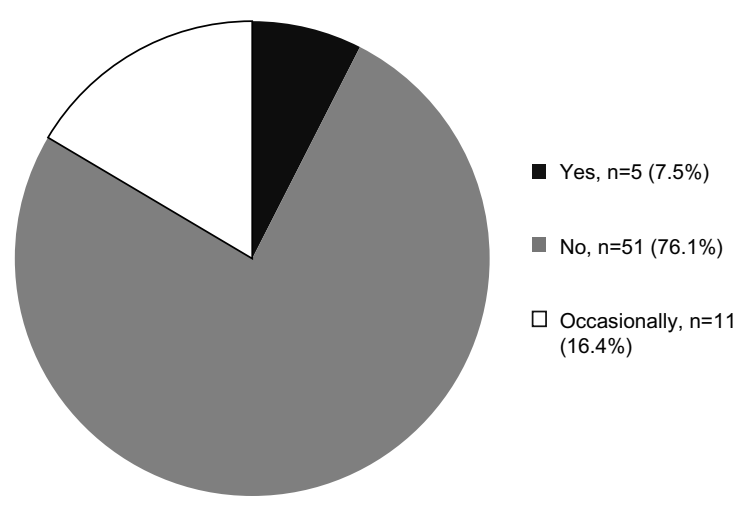

Figure 2 Responses to Q6: "Do you recommend/prescribe TENS to patients in pain at home?".

Abbreviation: TENS, transcutaneous electrical nerve stimulation.

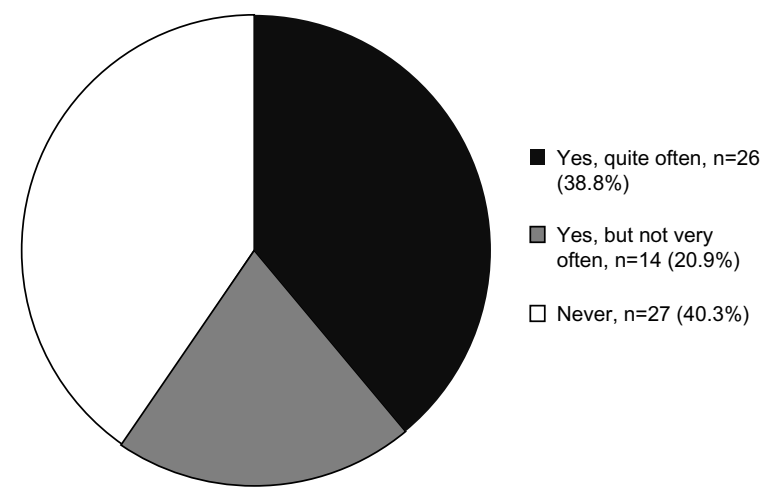

Figure 3 Responses to Q7: “Does your referring physician advise you to use TENS for pain relief?".

Abbreviation: TENS, transcutaneous electrical nerve stimulation.

reported that they often used TENS for neurological and musculoskeletal pain, but only "occasionally" for postsurgical pain and "seldom" for cancer pain. This pattern of use is similar to that seen in Australia, Europe, and USA, and is likely to reflect patient presentation patterns and beliefs of therapists about effectiveness of TENS for these conditions. ${ }^{8,25,26}$ Evidence from systematic reviews suggests that TENS is effective for chronic musculoskeletal pain, ${ }^{9}$ neuropathic pains, ${ }^{26,27}$ and postsurgical pain. ${ }^{10,28}$ Evidence for effectiveness for cancer pain is limited but promising. ${ }^{29}$

A main finding of the survey was that over $76.1 \%$ of respondents reported that they did not recommend that TENS should be used at home. Thus, patients would need to visit the clinic in order for each TENS treatment session. This is costly in staff time and inconvenient for the patient. More importantly, however, infrequent and short-duration TENS treatment is not optimal clinical practice. In most circumstances, it is recommended that TENS should be administered over the painful area or over peripheral nerves innervating the painful area.

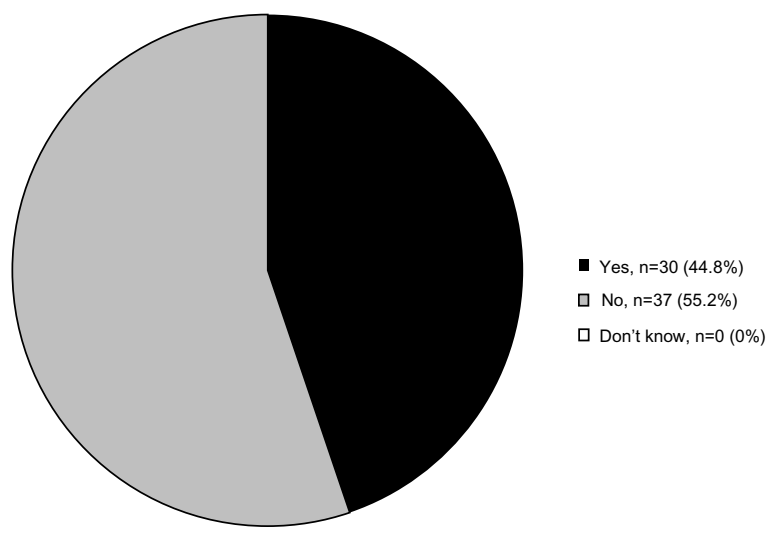

Figure 4 Responses to Q10: "Do you think TENS treatment is cost-effective compared with other treatments?".

Abbreviation: TENS, transcutaneous electrical nerve stimulation. 
Evidence suggests that a strong, non-painful TENS sensation is a prerequisite for success and that maximal pain relief occurs during stimulation. ${ }^{30}$ Therefore, successful long-term users of TENS self-administer TENS treatment regularly throughout the day because pain may return within minutes once TENS has stopped..$^{31}$ Unfortunately, the questionnaire did not include an item to gather information about why physiotherapists did not recommend that patients should self-administer TENS at home. One reason for this may be inappropriate beliefs about how best to administer TENS, perhaps resulting from inadequate undergraduate training. The small amount of training given on TENS and other electrotherapies in undergraduate training may contribute to the development of inappropriate beliefs about how best to administer treatment. Inappropriate beliefs that all electrotherapy treatments have to be supervised by a health care professional has been highlighted for interferential current therapy. ${ }^{32,33}$ Evidence suggests that patients can safely administer TENS themselves providing they are trained on safe and appropriate technique by a competent health care professional. ${ }^{31}$ Training protocols are readily available. ${ }^{34}$

Another reason why respondents did not recommend that TENS should be used at home may be that patients (and clinics) are unable to afford to purchase their own TENS device, including ongoing costs of replacing batteries and electrodes. This may account for the finding that only $44 \%$ of respondents reported that they believed that TENS was a cost-effective modality. One strategy to overcome this challenge is for patients to purchase or rent a TENS device from clinics or manufacturers at a subsidized cost with the proviso that the device is returned when it is no longer needed. This is an advantage of TENS when compared with drug medication, as the TENS device can be reused and recycled for use by other patients once it is no longer needed. The ability to finance the purchase of a sufficient stock of TENS devices to loan to patients may be a challenge for many clinics. Negotiating discounts for bulk purchase from manufacturers and charity fundraising activities can be used to reduce costs to the clinic. Furthermore, TENS equipment is considerably more expensive in Sri Lanka costing approximately 20000 Sri Lankan Rupees, equivalent to 9245 Indian Rupees. Indian-made TENS devices are available for approximately 2000 Indian Rupees, equivalent to 4330 Sri Lankan Rupees. Importing cheaper, Indian-made TENS devices in bulk may be a solution. Deterioration of electrodes due to dusty environments resulting in the need for more frequent replacement of electrodes may also increase running costs.

Sociocultural acceptance of TENS as a viable treatment option may also be a potential barrier to widespread use, including difficulties in securing the TENS device to clothes because of the absence of a belt, for example, for women who wear "sarees". Nevertheless, the finding that nearly half of respondents reported that their patients had requested TENS treatment suggests that individuals with pain attending physiotherapy clinics are aware of TENS, despite limited advertisement of TENS through mainstream media.

Sri Lanka faces considerable challenges in delivering consistent and quality health care services across the nation. ${ }^{21,22}$ Pain management has been described as a fundamental human right that should be available in primary care settings, ${ }^{34,36}$ yet pain management services in Sri Lanka are not given priority. ${ }^{35,36}$ Most pain management services are confined to large hospitals, often in the private sector, and located in major cities such as Colombo and Kandy. Provision in public or government hospitals appears to be inadequate. ${ }^{22}$ This restricts pain management to people who can afford to pay for expensive treatments. TENS may be a cost-effective option for managing pain because it is a safe, inexpensive, and effective treatment that can be self-administered by the patient as needed and without fear of toxicity or overdose.

There were some methodological limitations to this study. The survey sample size was small and restricted to urban centers. Secondary health care services such as physiotherapy are scantily resourced in rural Sri Lanka, so we suspect that TENS may be used less often in rural areas. The views of the $33 \%$ of physiotherapists that did not respond to the questionnaire are not known, and it is possible that these non-respondents did not use TENS or had used TENS previously and found it to be ineffective. For these reasons, we express caution in generalizing the findings to the wider physiotherapy population in Sri Lanka and we plan to conduct a larger in-depth survey of all health professionals engaged with TENS in rural as well as urban Sri Lankan areas.

\section{Conclusion}

Physiotherapists working in hospitals located in cities in Sri Lanka value TENS as a treatment option to manage musculoskeletal and neuropathic pain. They were less likely to use TENS for mild pain and for cancer pain. There appears to be a need for systems and resources to enable patients to selfadminister TENS rather than having to visit clinics.

\section{Acknowledgments}

The authors wish to acknowledge and thank physiotherapists of Teaching Hospital, Peradeniya; Kandy General Hospital, Kandy; National Hospital, Colombo; Nawaloka Hospital, Colombo; Central Hospital, Colombo; Lanka hospital, 
Colombo; Asiri Hospital, Colombo; Sulaiman's Hospital, Colombo; Hemas Hospital, Colombo; staff of the Department of Physiotherapy, Faculty of Allied Health Sciences, University of Peradeniya; International Research Center, University of Predaeniya; and, especially, Mr Sanjeewa Thunpattu, physiotherapist, National Hospital, Colombo, for their valuable support. The authors would like to thank the anonymous reviewers for their constructive advice.

\section{Author contributions}

TDD and MIJ conceived the study. TDD collected the data. TDD, GB, and MIJ performed the data analysis. All the authors contributed to the writing of the manuscript and revisions made following review. All authors read and approved the final manuscript.

\section{Disclosure}

The authors report no conflicts of interest in this work.

\section{References}

1. Gildenberg PL. History of electrical neuromodulation for chronic pain. Pain Med. 2006;7(Suppl 1):S7-S13.

2. Melzack R, Wall PD. Pain mechanisms: a new theory. Science. 1965;150(3699):971-979.

3. Shealy CN, Mortimer JT, Reswick JB. Electrical inhibition of pain by stimulation of the dorsal columns: preliminary clinical report. Anesth Analg. 1967;46:489-491.

4. Reynolds DV. Surgery in the rat during electrical analgesia induced by focal brain stimulation. Science. 1969;164:444-445.

5. Long DM. Electrical stimulation for relief of pain from chronic nerve injury. J Neurosurg. 1973;39:718-722.

6. DeSantana JM, Walsh DM, Vance C, Rakel BA, Sluka KA. Effectiveness of transcutaneous electrical nerve stimulation for treatment of hyperalgesia and pain. Curr Rheumatol Rep. 2008;10(6):492-499.

7. Jones I, Johnson MI. Transcutaneous electrical nerve stimulation. Continuing Education in Anaesthesia, Critical Care and Pain. 2009;9(4):130-135.

8. Johnson MI. Transcutaneous electrical nerve stimulation (TENS). In: Watson T, editor. Electrotherapy: Evidence-Based Practice. Edinburgh: Churchill Livingstone; 2008:253-296.

9. Johnson M, Martinson M. Efficacy of electrical nerve stimulation for chronic musculoskeletal pain: a meta-analysis of randomized controlled trials. Pain. 2007;130:157-165.

10. Bjordal JM, Johnson MI, Ljunggren AE. Transcutaneous electrical nerve stimulation (TENS) can reduce postoperative analgesic consumption. A meta-analysis with assessment of optimal treatment parameters for postoperative pain. Eur J Pain. 2003;7(2):181-188.

11. Dubinsky RM, Miyasaki J. Assessment: efficacy of transcutaneous electric nerve stimulation in the treatment of pain in neurologic disorders (an evidence-based review): report of the Therapeutics and Technology Assessment Subcommittee of the American Academy of Neurology. Neurology. 2010;74:173-176.

12. Rutjes AW, Nüesch E, Sterchi R, et al. Transcutaneous electrostimulation for osteoarthritis of the knee. Cochrane Database Syst Rev. 2009;(4): CD002823.

13. Johnson MI, Walsh DM. Pain: continued uncertainty of TENS' effectiveness for pain relief. Nat Rev Rheumatol. 2010;6: 314-316.
14. Bennett MI, Hughes N, Johnson MI. Methodological quality in randomised controlled trials of transcutaneous electric nerve stimulation for pain: low fidelity may explain negative findings. Pain. 2011;152(6): $1226-1232$.

15. Cameron MH, Lonergan E, Lee H. Transcutaneous electrical nerve stimulation (TENS) for dementia. Cochrane Database Syst Rev. 2003;(3):CD004032.

16. Cekmen N, Salman B, Keles Z, Aslan M, Akcabay M. Transcutaneous electrical nerve stimulation in the prevention of postoperative nausea and vomiting after elective laparoscopic cholecystectomy. J Clin Anesth. 2007;19(1):49-52.

17. Wikström SO, Svedman P, Svensson H, Tanweer AS. Effect of transcutaneous nerve stimulation on microcirculation in intact skin and blister wounds in healthy volunteers. Scand J Plast Reconstr Surg Hand Surg. 1999;33(2):195-201.

18. Elzahaf RA, Tashani OA, Unsworth BA, Johnson MI. The prevalence of chronic pain with an analysis of countries with a Human Development Index less than 0.9: a systematic review without meta-analysis. Curr Med Res Opin. 2012;28(7):1221-1229.

19. Census of Population and Housing - 2012 [webpage on the Internet]. Population Distribution - 2012. Department of Census and Statistics Sri Lanka. Available from: http://www.statistics.gov.lk/PopHouSat/ CPH2012Visualization/htdocs/index.php? usecase=indicator\&action= Map\&indId=10). Accessed April 20, 2014.

20. IASP-pain.org [homepage on the Internet]. Unrelieved pain is a global healthcare problem. Available from:http://www.iasp-pain.org/files/ Content/ContentFolders/GlobalYearAgainstPain2/20042005RighttoP ainRelief/factsheet.pdf. Accessed April 20, 2014.

21. Perera M, Gunatilleke G, Bird P. Falling into the medical poverty trap in Sri Lanka: what can be done? Int J Health Serv. 2007;37(2): 379-398.

22. Somanathan A, Hanson K, Dorabawila T, Perera B. Operating Efficiency in Public Sector Health Facilities in Sri Lanka: Measurement and Institutional Determinants of Performance. Small Applied Research Paper No 12. Available from: http://rep216.infoeach.com/ view-MjE2fDkzODg1OA==.html. Accessed April 20, 2014.

23. Gureje O, Von Korff M, Simon GE, Gater R. Persistent pain and well-being: a World Health Organization study in Primary Care. JAMA. 1998;280(13):1142.

24. Banerjee G, Johnson MI. A survey of physiotherapists' attitudes and beliefs about the use of TENS for pain management in India. International Journal of Scientific Research and Reviews. 2013;2(3):36-46.

25. Chipchase LS, Williams MT, Robertson VJ. A national study of the availability and use of electrophysical agents by Australian physiotherapists. Physiother Theory Pract. 2009;25(4):279-296.

26. Johnson MI, Bjordal JM. Transcutaneous electric nerve stimulation for the management of painful conditions: focus on neuropathic pain. Expert Rev Neurother. 2011;11(5):735-753.

27. Jin DM, Xu Y, Geng DF, Yan TB. Effect of transcutaneous electrical nerve stimulation on symptomatic diabetic peripheral neuropathy: a meta-analysis of randomized controlled trials. Diabetes Res Clin Pract. 2010;89:10-15.

28. Freynet A, Falcoz PE. Is transcutaneous electrical nerve stimulation effective in relieving postoperative pain after thoracotomy? Interact Cardiovasc Thorac Surg. 2010;10(2):283-288.

29. Hurlow A, Bennett MI, Robb KA, Johnson MI, Simpson KH, Oxberry SG. Transcutaneous electric nerve stimulation (TENS) for cancer pain in adults. Cochrane Database Syst Rev. 2012;3:CD006276.

30. Moran F, Leonard T, Hawthorne S, et al. Hypoalgesia in response to transcutaneous electrical nerve stimulation (TENS) depends on stimulation intensity. J Pain. 2011;12(8):929-935.

31. Johnson MI, Ashton CH, Thompson JW. An in-depth study of longterm users of transcutaneous electrical nerve stimulation (TENS). Implications for clinical use of TENS. Pain. 1991;44(3):221-229.

32. Johnson M. The mystique of interferential currents. Physiotherapy. 1999;85(6):294-297. 
33. Tabasam G, Johnson MI. The use of interferential therapy for pain management by physiotherapists. Int J Ther Rehabil. 2006;13(8):357-364.

34. Johnson MI. Transcutaneous Electrical Nerve Stimulation (TENS). Research to Support Clinical Practice. Oxford: Oxford University Press; 2014.
35. Brennan F, Carr DB, Cousins M. Pain management: a fundamental human right. Anesth Analg. 2007;105(1):205-221.

36. Imani F, Safari S. "Pain Relief is an Essential Human Right", We Should be Concerned about It. Anesth Pain. 2011;1(2):55-57.

Patient Related Outcome Measures

\section{Publish your work in this journal}

Patient Related Outcome Measures is an international, peer-reviewed, open access journal focusing on treatment outcomes specifically relevant to patients. All aspects of patient care are addressed within the journal and practitioners from all disciplines are invited to submit their work as well as healthcare researchers and patient support groups.
The manuscript management system is completely online and includes a very quick and fair peer-review system. Visit http://www.dovepress. com/testimonials.php to read real quotes from published authors. 